\title{
LASIK surgery in pediatric anisometropia
}

\author{
Hany M. Bayoumy, Ihab A. El-shaikh, Nour-eldeen A. Salah * \\ Department of Ophthalmology, Faculty of Medicine, Al-Azhar University, Cairo, Egypt \\ *Corresponding author: Nour-eldeen A. Salah, E-mail: drnourahmed1988@gmail.com, Telephone No.:+2001067833922
}

\begin{abstract} when conventional therapies had failed. laser in-situ keratomileusis (LASIK) surgery. corneal topography and complications were evaluated. three months after surgery. children.

\section{INTRODUCTION}

Anisometropia is the most common cause of amblyopia, which occurs due to uncorrected asymmetrical refractive error between the two eyes. Uncorrected anisometropia induces blurry image in one eye and unusual binocular interaction by producing different images on the fovea ${ }^{(\mathbf{1})}$. In recent years, a number of possible surgical procedures in ophthalmology has offered prospective patients an alternative to wear spectacles or contact lenses ${ }^{(2)}$. Methods that may be useful in children are Photorefractive keratectomy (PRK), LASIK and laser supepithelial keratomileusis (LASEK). PRK and LASIK have been the most widely studied among the excimer laser procedures.
\end{abstract}

Background: Refractive surgery, particularly laser in-situ keratomileusis (LASIK) surgery, at present offers hope in pediatric anisometropia, particularly where traditional therapy has failed. Studies show that it is effective and safe procedure that corrects high anisometropia and improved binocularity,

Aim of the Work: to evaluate the visual outcomes of patients with anisometropic amblyopia after

Patients and Methods: this study enrolled 20 anisometropic eyes of 20 patients ranging from 6 to 12 years with refractive difference between two eyes more than four diopters, ,LASIK was performed in the amblyopic eye under topical or general anaesthesia according to cooperation of the patients. Visual acuity, refraction,

Results: The mean preoperative spherical equivalent refraction (SE) in the operated eye was $-5.89 \pm 1.46$ diopter (range: -10.00 to $-4.24 \mathrm{D}$ ), significantly decreased down to $-0.36 \pm 0.19 \mathrm{D}$ at one week, $-0.38 \pm 0.19 \mathrm{D}$ at one month, $0.46 \pm 0.26 \mathrm{D}$ at three months postoperatively. The mean uncorrected visual acuity in the operated eye was $0.58 \pm 0.13$ (range: 0.30_A0.8) gained five or more lines on snellens chart. The best corrected visual acuity also shows a great improvement in comparing to the preoperative state by one or more lines

Conclusion: LASIK is effective, safe and predictable alternative method for correcting anisometropia in

Keywords: pediatric anisometropia, amblyopia, laser in situ keratomileusis.

\section{AIM OF THE WORK}

To evaluate the visual outcomes of patients with anisometropic amblyopia after (LASIK) surgery.

\section{PATIENTS AND METHODS}

This study included 20 anisometropic eyes of 20 patients with refractive difference between two eyes more than four diopters, ranging from 6 to 12 years old had undergone LASIK surgery at many LASIK centers. The study protocol was adhered to the tenets of the declaration of Helsinki ${ }^{(3)}$ and was approved by the Ethics Board of AlAzhar University. An informed written consent was taken from each participant in the study.
Every patient in this study was subjected to the following:

i-History taking from parents including:Age, sex, previous ocular problems or systemic health problems and Family history.

ii-Routine ophthalmic examination including Visual acuity (VA): uncorrected Visual acuity (UCVA) and best corrected Visual acuity (BCVA) using Snellen's chart , Refraction: Manifest refraction: equals to correction with the most accepted power that gives the BCVA,

Cycloplejic refraction:cyclopentolate hydrocloride $1 \%$ eye drops was installed six drops 10 minutes apart.cycloplegic refraction used as a base to surgical plan , and to compare preoperative and postoperative results, anterior segment evaluation by slit lamp ,corneal topography using pentacam or orbscan, fundus evaluation by indirect ophthalmoscope and treatment of amblyopia using part time occlusion regimn .

The subjects included in this study were patients from 6 to 12 old years with clinical features of anisometropia more than four diopters and limited refraction in the sound eye less than - 10 diopters with normal retinae and lenses .

The exclusion criteria included the presence of congenital cataract, corneal opacities.associated 
posterior segment pathology, abnormal corneal topography or active inflammation.

Surgical procedure: Topical anaethesia using Benoxinate hydrochloride $0.4 \%$ eye drops was installed preoperatively one drop three times five minute apart for 9 cooperative patients and general anaethesia was used for 11 uncooperative patients. Allegretto EX500 excimer laser was used and laser calibration was checked before the procedure.

Demographic data (name,sex,age) and referactive data were intered into the computer,optical zone diameter was 6.0 to $6.5 \mathrm{~mm}$ according to the pupil size,thickness of the cornea and the error of referaction and preoperative keratometry.

Awire lid speculum was put in place . A corneal marking was used which helps in reposition of the corneal flap at the end of procedure. The patient was insructed to fix his eye and to maintain fixation to a flickering green light.The suction ring was centered and the suction was activated.Elevation in the intraocular pressure up to $60 \mathrm{~mm} \mathrm{Hg}$ was checked by the surgical tonometer. the patient was informed that the flickering light would disappear.The microkeratome head was engaged to the ring. The forward pedal had been pressed to make a soperior hanged corneal flap , and then the reverse pedal was pressed to get it back. During this step patient was informed that there will be a period of non-seeing and should be assured that this is normal.

The microkeratome head was disengaged, the suction was stopped and the ring was removed.

The flap was folded back with a canula. Excimer laser ablation was focused and carried out in the stromal bed.The surface of the bed and the flap were moistened and irregated with balanced salt solution, and the flap was folded back onto the cornea using a cannula.

The edge of the flap was dried with microsponges to ensure good adhesion between the flap and the bed.Removal of the lid speculum .patient instructed to blink quickly and gently.Contact lenses were used for 11 eyes for 3 days. Patients were examined half an hour after procdure to assess positioning of the flap, regularity of the surface,edge of the flap and interface deposits. After the operation all patients received the same standard medication, consisting of combined (steroid and antibiotic) eye drops had used five times per day for five days, a tear replacement eye drops were used six times per day for a three months,a clear plastic eye shield was used to cover the eye for 3 days postoperatively and a treatment of amblyopia using part time occlusion regimen was continued post-surgery.

\section{FOLLOW UP}

Patients were examined on Slit lamp biomicroscopy half an hour after procedure ,first postoperative day ,one week,one month and three months
.Refraction:Maniffest and cycloplejic refraction were done postoperatively at the end of the first week, the first month and the third month postoperatively to be compared with the preoperative refraction.Uncorrected and best corrected visual acuity.Corneal topography was done after Three months postoperatively to show the amount of the steepening or flattening and to show differences between pre and postoperative values.

\section{RESULTS}

This study included 20 eyes of 20 patiants. There were 8 males ( 8 eyes, $40 \%$ ) and 12 females (12 eyes $60 \%$ ). patient's age ranged from 6to 12 years, the mean age was $[8.45 \pm 1.96]$ years. All patients had unilateral surgery. 9 patients ( $45 \%$ ) had surgery for the rright eye, and 11 patients (55\%) had surgery in the left eye.The mean preoperative spherical equivalent refraction (SE) in the operated eyes was $-5.89 \pm 1.46$ diopter (range: -4.25 to $-10.00 \mathrm{D}$ ), Postoperatively, there was a great reduction in the mean spherical equivalent down to $-0.36 \pm 0.19$ diopter (range: 0.00 to $-0.75 \mathrm{D}$ ) one week after surgery.One month after surgery the mean spherical equivalent refraction (SE) in the operated eyes was $-0.38 \pm 0.19$ diopter (range:0.00 to $-0.75 \mathrm{D}$ ). Three months after surgery the mean spherical equivalent refraction (SE) in the operated eyes was $-0.46 \pm 0.26$ diopter (range: 0.25 to $-0.75 \mathrm{D}$ ). Four patients ( $20 \%)$ had a significant cylindrical refraction (range: - 1.00 to-2.00 D) complete improvement had occurred in one patient and slight under correction occurred with other three patients ranging postoperatively from -0.75 to $-1.25 \mathrm{D}$. Table (1) and Fig.(1): show highly statistically significant improvement between preoperative and postoperative spherical equivalent (Diopter).

Table (1): The extent of the difference over the periods through refraction: spherical equivalent (diopter) in the study group.

\begin{tabular}{|l|l|l|}
\hline $\begin{array}{l}\text { Refraction: } \\
\text { Spherical } \\
\text { equivalent } \\
\text { (Diopter) }\end{array}$ & Range & Mean \pm SD \\
\hline Pre-operative & $-4.25 \_-10.00$ & $-5.89 \pm 1.46$ \\
\hline After 1st week & $0.00 \_-0.75$ & $-0.36 \pm 0.19$ \\
\hline After 1stmonth & $0.00 \_-0.75$ & $-0.38 \pm 0.19$ \\
\hline After 3rdmonths & $-0.25 \_-0.75$ & $-0.46 \pm 0.26$ \\
\hline
\end{tabular}


Hany Bayoumy et al.
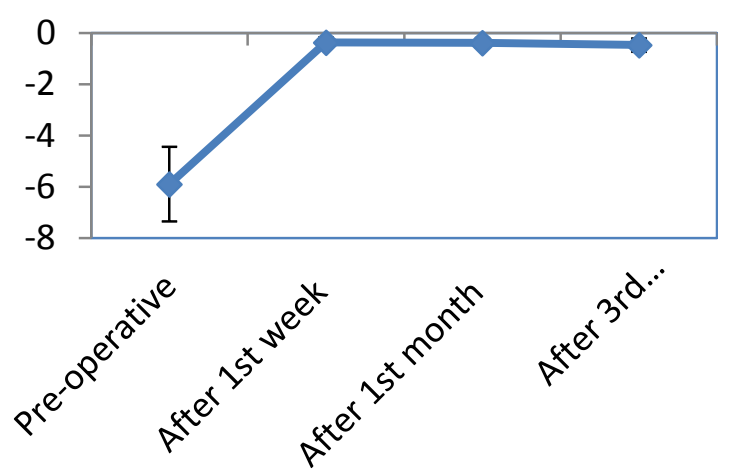

Fig.(1): Line shows of the difference over the periods through refraction: spherical equivalent (diopter) in the study group.

The mean preoperative uncorrected visual acuity in the operated eyes was $0.05 \pm 0.03$ (range 0.01_0.08), Postoperatively, there was a great Improvement in the mean uncorrected visual acuity in the operated eyes up to $0.50 \pm 0.12$ (range: $0.30 \_0.6$ ) one week after surgery. One month after surgery The mean uncorrected visual acuity in the operated eyes was $0.54 \pm 0.12$ (range: 0.30_A0.7).Three months after surgery the mean uncorrected visual acuity in the operated eyes was $0.58 \pm 0.13$ (range: $0.30 \_A 0.7$ ). Table (2) and Fig.(2): show highly statistically significant difference between preoperative with one week,one month, and three months postoperatively according to uncorrected visual acuity.

Table (2): The extent of the difference over the periods through uncorrected visual acuity in the study group.

\begin{tabular}{|l|l|}
\hline $\begin{array}{l}\text { Uncorrected visual } \\
\text { acuity }\end{array}$ & Range \\
\hline Pre-operative & $0.01 \_A 0.08$ \\
\hline After 1st week & $0.30 \_A 0.6$ \\
\hline After 1st month & $0.30 \_A 0.7$ \\
\hline After 3rd months & $0.30 \_A 0.7$ \\
\hline
\end{tabular}

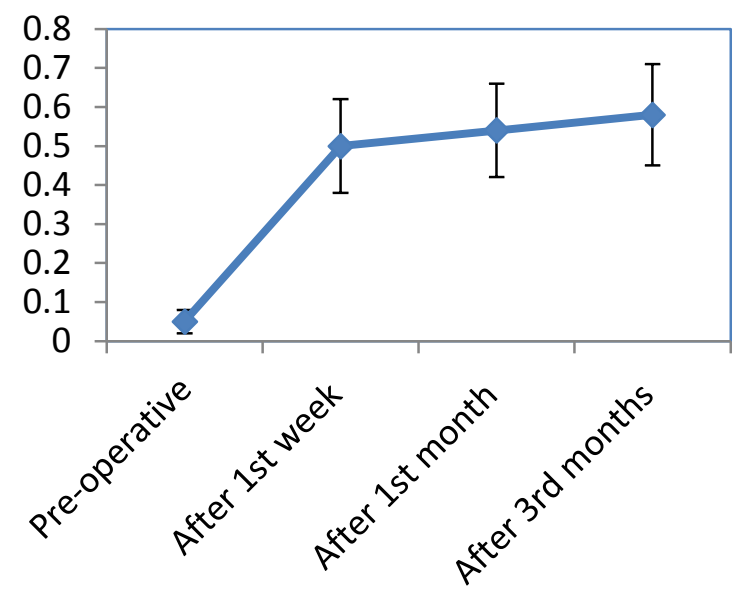

Fig. (2): Line shows of the extent of the difference over the periods through uncorrected visual acuity in the study group.
The mean preoperative best corrected visual acuity in the operated eyes was $0.51 \pm 0.13$ (range 0.30_A0.60), one week after surgery the mean best corrected visual acuity in the operated eyes was $0.51 \pm 0.12$ (range: 0.30_0.7).one month after surgery there was a great Improvement in the mean best corrected visual acuity in the operated eyes up to $0.57 \pm 0.11$ (range: $0.40 \_$A0.7).Three months after surgery the mean best corrected visual acuity in the operated eyes was still improving up to $0.63 \pm 0.14$ (range: 0.40_A0.9). Table (3): shows highly statistically significant difference between preoperative and one week with one month, and three months postoperatively according to the best corrected visual acuity. Fig.(3):Line shows the difference over the periods through the best corrected visual acuity in the study group.

Table (3): The extent of the difference over the periods through best corrected visual acuity in the study group.

\begin{tabular}{|l|l|}
\hline $\begin{array}{l}\text { Best corrected } \\
\text { visual acuity }\end{array}$ & Range \\
\hline Pre-operative & $0.30 \_A 0.6$ \\
\hline After 1st week & $0.30 \_A 0.7$ \\
\hline After 1st month & $0.40 \_A 0.7$ \\
\hline After 3rd months & $0.40 \_A 0.9$ \\
\hline
\end{tabular}

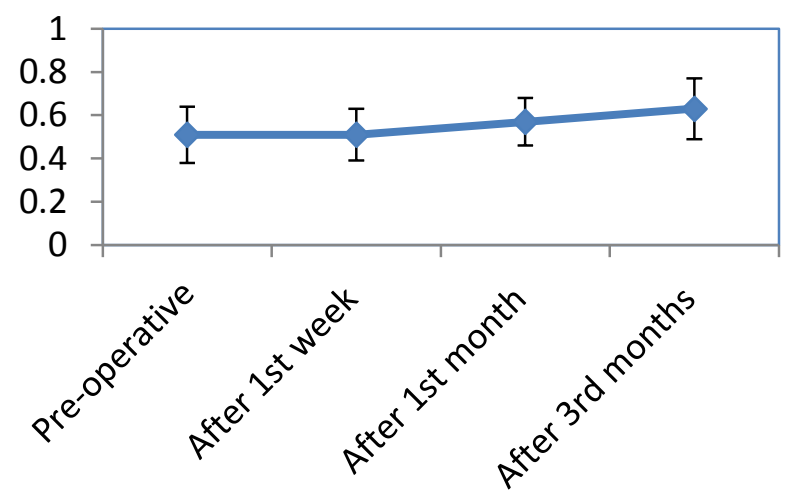

Fig.(3):Line shows of the extent of the difference over the periods through best comected visual acuity in the study group.

During surgery none of the eyes suffered from sight threating complications.there were no serious complications related to microkeratome.

There were no free caps, and no damage or destruction to the flaps.

Two patients suffered from postoperative pain which disappeared by the second day, Three patients had flap displacement ,five patients had postoperative mild residual refraction (range - 0.25 to -0.75$)$. 


\section{DISCUSSION}

Anisometropia is one of the most common causes of amblyopia. Improved vision in the amblyopic eye by spectacles is usually associated with intolerable aniseikonia and may be diplopia ${ }^{(4,5)}$.

Contact lenses provided a better visual quality, a larger visual field and improved contrast sensitivity (6).

However, long-term use of contact lenses has potential infection risks and it is also not feasible for children.Therefore, the effect of traditional therapy for these patients is often poor.

Recently some researchers began to explore the application of LASIK in the treatment of anisometropia ${ }^{(7,8,9,10,1)}$.

Many researchers studied LASIK for correction of anisometropia in children.Their results regarding refraction, the visual acuity, safety and efficacy will be discussed and points of differences and similarities between them and our study will be discussed.

The treatment goal in those children is to improve their UCVA, which can enhance their social development and improve their school performance.

In the past, there had been no effective treatment for this group of children. LASIK is a viable method for correcting ametropia with few complications.

In our study, we evaluated the visual outcome of 20 patients with anisometropic amblyopia after LASIK surgery.The mean age of the patients was 6-12 years (8.45 \pm 1.96$)$.

The mean manifest spherical equivalent refraction of the treated eyes was

$-5.89 \pm 1.46 \mathrm{D}$ preoperative and showed a marked reduction to became

$-0.46 \pm 0.26 \mathrm{D}$ postoperatively.

The preoperative mean UCVA \pm SD was $0.05 \pm 0.03$ and became $0.58 \pm 0.13$, whereas the preoperative mean BCVA was $0.51 \pm 0.13$ and became $0.63 \pm 0.14$ 3 months postoperatively.

Uncorrected visual acuity of all patients improved significantly about five or more lines in Snellen's chart. The BCVA of all patients improved by one or more lines in Snellen's chart.

There were no serious complications in our study, none of the eyes suffered from sight threating complications during surgery.

There were no complications related to microkeratome (no free caps, and no damage or destruction to the flaps).

In the study of Lin et al. ${ }^{(9)}$. They concluded that LASIK in treatment of high pediatric anisometropia with monocular amblyopia may be effective and safe.

Their study was performed on 24 eyes (five myopes and 19 hypermetropes) with high unilateral anisometropic amblyopia, who underwent LASIK . The mean age of these children was $7.4 \pm 1.9$ years and ranged from 5 to 14 years. The mean preoperative UCVA was $0.06 \pm 0.05$ and was improved significantly postoperative to $0.43 \pm 0.33$ , whereas the mean preoperative BCVA was $0.26 \pm$ 0.22 and was improved postoperatively to $0.67 \pm$ 0.40 . The mean myopic spherical equivalent refraction was $-8.01 \pm 2.70 \mathrm{D}$ for myopic anisometropic patients, whereas the postoperative value reduced significantly to $-1.32 \pm 2.47 \mathrm{D}$.

For patients with hyperopic anisometropia, the preoperative mean spherical equivalent refraction was $+7.35 \pm 1.55 \mathrm{D}$, whereas the postoperative value reduced significantly to $+3.30 \pm 0.86 \mathrm{D}$.

Assad et al. ${ }^{(10)}$.Evaluated the effects of LASIK in treatment of myopic anisometropia in 25 children, with mean spherical equivalent refraction in the amblyopic eye had reduced significantly from -9.08 $\pm 1.86 \mathrm{D}$ preoperatively to $-0.97 \pm 1.16 \mathrm{D}$ at two years postoperatively.

The preoperative mean spherical equivalent myopic anisometropia was $-7.75 \pm 2.25 \mathrm{D}$ and $-0.50 \pm 0.21$ $\mathrm{D}$ at two years postoperatively, representing a 93.5\% reduction in anisometropia.

Utine et $\boldsymbol{a l}^{(12)}$. Evaluated the results of LASIK for hypropia in pediatric anisometropic amblyopia.

This study included 32 children. The mean follow up was $20.1 \pm 15.1$ months (range 12 to 60 months). The mean preoperative SE refraction had been $+5.17 \pm 1.65 \mathrm{D}$ and the mean postoperative SE refraction was $+1.39 \pm 1.21 \mathrm{D}$.

Comparing their results with those of our study, our patients had a lower age range of 6-12 years. The mean preoperative UCVA in our study was slightly lower than that in the other studies. It was $0.05 \pm 0.03$ preoperatively and was elevated postoperatively to $0.56 \pm 0.18$ which is also higher than their results. Our mean preoperative BCVA was $0.39 \pm 0.13$ and was elevated postoperatively to $0.58 \pm 0.13$ which was also higher than the Lin and colleagues's result.

The preoperative and postoperative mean spherical equivalent refraction was lower than in the Lin and colleagues's and A ssad et al studies.

Comparing their result in hypropic anisometropic eyes with those of our study in myopic anisometropia, The postoperative mean spherical equivalent refraction in our patients had higher signifecant improvement. 
Dedhia and Behl ${ }^{(13)}$. Evaluated the efficacy and benefit of LASIK in anisometropic amblyopia.

21 eyes of 21 anisometropic amblyopes with a mean age of $27.71 \pm 12.24$ years had undergone LASIK. 3 patients were less than 18 years of age and the follow up period was three months, whereas in our study, the mean age of the patients was lower (6-12 $[8.45 \pm 1.96]$ years) and the follow-up period was three months.

The mean manifest spherical equivalent refraction in their study was $-13.20 \pm 3.84 \mathrm{D}$ preoperatively, and postoperative it significantly reduced to -0.78 $\pm 1.63 \mathrm{D}$. The postoperative UCVA improved by two or more lines in all patients.

It was the same as or better than the preoperative best spectacle-corrected visual acuity (BSCVA) in $95.2 \%$ of the eyes. In our study, the mean manifest spherical equivalent refraction $-5.89 \pm 1.46 \mathrm{D}$ and became $-0.46 \pm 0.26 \mathrm{D}$ postoperatively.

The postoperative UCVA also improved by 5 or more lines in all patients and was the same as or better than the preoperative BSCVA in all of the patients.

Comparing their result in adults with those of our study in children, our patients had a lower age range of 6-12 years. The pre and postoperative mean spherical equivalent refraction was lower than in the Dedhia and Behl study .our patients had higher signifecant improvement in UCVA postoperatively.

Astle et al. ${ }^{(8)}$. Have reported the safety and effectiveness of LASIK for the correction of anisometropia in children. It has shown that this surgery can effectively improve visual acuity and reduce refraction with no more complications than would be expected in adults.

Phillips et al. ${ }^{(14)}$. Have studied LASIK for the correction of anisometropia in amblyopic children patients who completed amblyopia therapy, have an average anisometropia of $4.43 \mathrm{D}$ bilaterally and a visual acuity of $6 / 9$ or better bilaterally at an average 18-months follow-up.

Agrawal et al. ${ }^{(15)}$. Reported 12 eyes regained their best corrected visual acuities in 16 anisometropic amblyopic eyes in pediatric age group, and they think some cases of anisometropic amblyopia when other treatments failed are encouraged to receive LASIK.

In our study patients had a great significant improvement in UCVA and BCVA . the mean manifest spherical equivalent showed a marked reduction from $-5.89 \pm 1.46 \mathrm{D}$ preoperatively to $0.46 \pm 0.26 \mathrm{D}$ postoperatively, with no reduction in UCVA or BCVA, and none of the eyes in our study suffered from sight threatening complications during or after surgery.

There were no complications related to microkeratome (no free caps, and no damage or destruction to the flaps).

\section{CONCLUSION}

All these results with our result demonstrate that LASIK is safe, effective and predictable alternative method for correcting anisometropia facilitating treatment of amblyopia, not responding to conventional methods of treatment .Further clinical studies with longer follow-up and larger series are essential to establish the long-term efficacy and safety of LASIK surgery in very young pediatric patients.

\section{REFERENCES}

1. De Vries J (1985): Anisometropia in children: analysis of a hospital population. $\mathrm{Br} \mathbf{J}$ Ophthalmol.,1985,69:504-508.

2. D'Arcy FM, Kirwan C, O'keefe M(2012): Ten year follow up of laser in situ keratomileusis for all levels of myopia. Acta Ophthalmol.,90:e335-6.

3. World Medical Association Declaration of Helsinki(2013): Ethical Principles for Medical Research Involving Human Subjects.JAMA., 310(20):2191-2194.

4. Cobb DJ, Russell K, Cox A(2002) Factors influencing visual outcome in anisometropic amblyopes. Br J Ophthalmol., 86 :1278-1281.

5. Woodruff G, Hiscox F, Thompson JR et al. (1994): The presentation of children with amblyopia. Eye, $8: 623-626$.

6. Freeman M(1992): Spectacles vs contact lenses in the correction of unilateral axial myopia. Arch Ophthalmol., $110: 180$.

7. Yin ZQ, Wang H, Yu T et al.(2007): Facilitation of amblyopia management by laser in situ keratomileusis in high anisometropic hyperopic and myopic children. J AAPOS.,11 :571-576.

8. Astle WF, Rahmat J, Ingram AD et al.(2007): Laser-assisted subepithelial keratectomy for anisometropic amblyopia in children. J Cataract Refract Surg., 33 :2028-2034.

9. Lin XM, Wang Z, Yang B et al.(2009): Longterm efficacy of excimer laser in situ keratomileusis in the management of children with high anisometropic amblyopia. Chin Med J., 122:813-817.

10. Assad AG, Ashraf IM, Ehab HN et al.(2010): Laser in situ keratomileusis for treated myopic anisometropic amblyopia in children. Saudi J Ophthalmol., 24 :3-8. 
11. Tamer AR, Olfat AH (2011): Evaluation of improvement of best corrected visual acuity following LASIK treatment in anisometropic amblyopia. Aust J Basic Appl Sci., 5:23-29.

12. Utine CA,Cakir H, Egemenoglu et al.(2008): LASIK in children with hypropic anisometropic amblyopia. J Refract Surg., 24(5):46-72.
13. NC Dedhia, S Behl.(2000): Laser in situ keratomileusis for anisometropic amblyopia. J Refract Surg, 16(1) :S264-S267.

14. Phillips C, Prager et al.(2004): Laser in situ keratomileusis for treated anisometropic amblyopia in awake, autofixating pediatric and adolescent patients. J. Cataract Refract. Surg., 30: 2522-2528.

15. Agrawal A, Agrawal et al .(2000): Results of pediatric laser in situ keratomileusis. J.Cataract Refract. Surg., 26:684-689. 